\title{
Coupled-Mode Flutter of a Cleaning Blade System in a Laser Printer"
}

\author{
Minoru KASAMA $^{* *}$, Masatsugu YOSHIZAWA ${ }^{* * *}$, Yimei YU ${ }^{* *}$, Tomoyuki $_{\text {ITOH }}{ }^{* *}$ \\ and Yoshiaki ITOH $^{* * * *}$ \\ ** Dept. of Key Technologies Development, Fuji Xerox Co., Ltd., \\ Sakai 430, Nakai-cho, Ashigarakami-gun, Kanagawa 259-0157, Japan \\ E-mail: minoru.kasama@fujixerox.co.jp \\ *** Dept. of Mechanical Engineering, Keio University, \\ Hiyoshi3-14-1, Kohoku-ku, Yokohama City, Kanagawa 223-8522, Japan \\ E-mail: dynamics@mech.keio.ac.jp \\ ****Dept. of Commerce Industry and Labor, Kanagawa Prefectural Government, \\ Nihon-odori 1, Naka-ku, Yokohama City, Kanagawa 231-8588, Japan \\ E-mail: itoh@kanagawa-iri.go.jp
}

\begin{abstract}
This paper discusses a mechanism of a self-excited vibration of a cleaning blade in a laser printer. We present a coupled-mode flutter model using a finite element model. A stability analysis based on the proposed model is carried out. From this result, it is clarified that two modes couple each other with increasing coefficient of friction. At that time, the natural frequency of the coupled-mode is corresponding to the frequency of the self-excited vibration. This root locus reveals a typical argand diagram for coupled-mode flutter of an undamped system via so-called Hamiltonian-Hopf bifurcation. Furthermore, we discuss about the steady-state amplitude of the self-excited vibration. First, we present a nonlinear amplitude equation by extracting the unstable modes with introduction of the adjoint vector to the eigenvector of the system. Second, from consideration about the nonlinear term which is able to restrain an increasing of amplitude, we decide the nonlinear term referring to the Rayleigh's equation. Then, the unstable mode solution obtained by the method of multiple scales is reconstructed in 2-DOF system by referring to Herrmann and Roussellet's method in pipes conveying fluid. Finally, we present the theoretical equation of the steady-state amplitude. We reveal a validity of our study by a comparison between an experiment and a numerical simulation of some modified blades.
\end{abstract}

Key words: Coupled-Mode Flutter, Frictional Vibration, Information Processing Equipment, Cleaning Blade, Hamiltonian-Hopf Bifurcation, Nonlinear Amplitude Equation

\section{Introduction}

It is very important to avoid occurrence of uncomfortable noise, since laser printers have many opportunities to be used near users. In this paper, we discuss a mechanism of a self-excited vibration of a cleaning blade in a laser printer. Up to now, there are some conventional studies about the squeaking noise due to a self-excited vibration in a copy machine or a printer. Nakamura ${ }^{(1)}$ clarified the mechanism that frictional force excites a twist vibration mode of a photoreceptor drum. Kawamoto ${ }^{(2)}$ focused a squeaking noise occurring just after starting of a drum rotation or just before stopping. The mechanism was explained as a nonlinear phenomenon combined a self-excited vibration due to a negative 
damping of dry friction and a forced vibration by a bias charger roller.

In this paper, we present a coupled-mode flutter model using finite element model. By using the presented model, a stability analysis is carried out on a numerical simulation, we reveal results as follows: 1) Two modes couple each other with increasing coefficient of friction; 2) The natural frequency of the coupled-mode is corresponding to the frequency of the squeaking noise when the system becomes unstable. Next, in order to extract only unstable modes, we introduce an eigenvector of the adjoint system. It is shown that this method is effective to solve the non-orthogonality between eigenvectors of the original system due to the nonsymmetrical stiffness matrix. Besides, we determine a nonlinear term from the condition to avoid an appearance of a secular term and formulate a nonlinear equation about unstable mode displacement. Furthermore, the solution of unstable mode displacement obtained by the method of multiple scales is reconstructed in 2-DOF system by utilizing of the method of Herrmann and Roussellet ${ }^{(3)(4)}$. Then, we express a displacement of the cleaning blade by using the eigenfunction of non-coupled system and unstable mode displacement. Finally, the validity of our study is verified by the comparison between an experiment and a numerical simulation of some modified blades.

\section{Cleaning Blade Model}

\subsection{Observation in an Actual Machine}

Figure1 shows a schematic diagram of a toner cartridge in a laser printer. The cleaning blade consists of a gum blade and a steel plate. It is fixed on the housing frame. In order to clean up the photoreceptor surface, the cleaning blade is pressed on the photoreceptor in the counter direction to the photoreceptor rotation. The self-excited vibration occurs at the part where the cleaning blade contacts with the photoreceptor. Figure 2 shows the frequency spectrum of the self-excited vibration at the edge of a cleaning blade in an actual machine by a Laser Doppler Vibrometer (LV-1100 ONO SOKKI) through a hole cut on the housing frame. The main frequency of this self-excited vibration is $4588 \mathrm{~Hz}$, and the amplitude is converged to a non-trivial value after time passes.

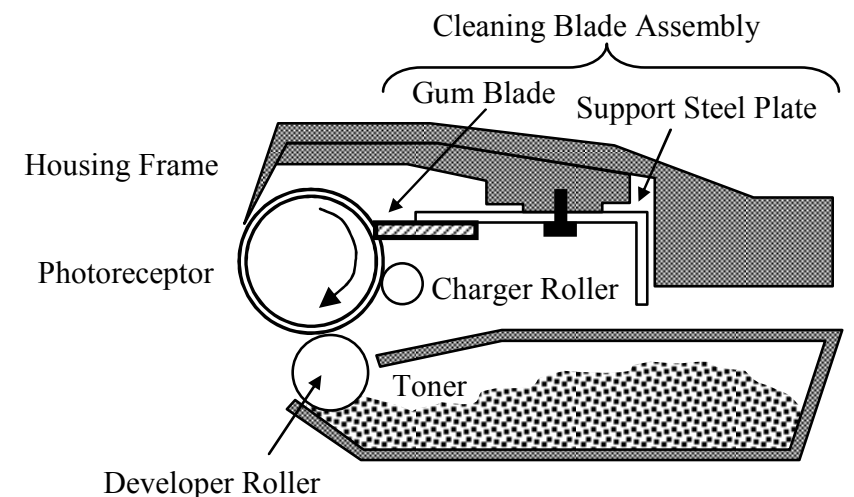

Fig. 1 Schematic diagram of toner cartridge in a laser printer.

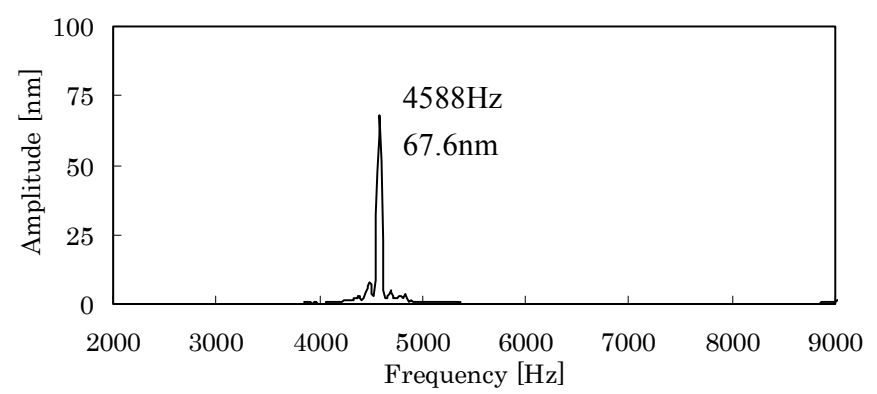

Fig. 2 Observation result of vibration at the blade edge in an actual machine 


\subsection{Finite Element Model of the Cleaning Blade}

In order to clarify the mechanism of the self-excited vibration, we direct our attention to a coupled-mode flutter model. This model has already been applied to the self-excited vibration of a wiper system ${ }^{(5)}$ or a contact head slider in a magnetic recording equipment ${ }^{(6)}$.

We consider a finite element model of the cleaning blade as shown in Fig. 3. The design parameters of the cleaning blade are shown in Table 1. The finite element package ANSYS has been used to carry out the finite element analysis in this study. The meshes were constructed using 2-D four-node quadrilateral element (PLANE42) by the mapped mesh function of ANSYS. In this case, 301 nodes have been generated. The blade and the photoreceptor contacts at only one point (Node Number 1) each other. Furthermore, we created a contact element (CONTAC171) and a target element (TARGE169) at the contact point to impose the limitation that the blade did not part from the photoreceptor. The displacement of the nodes fixed to the support steel plate was restrained to 0 .

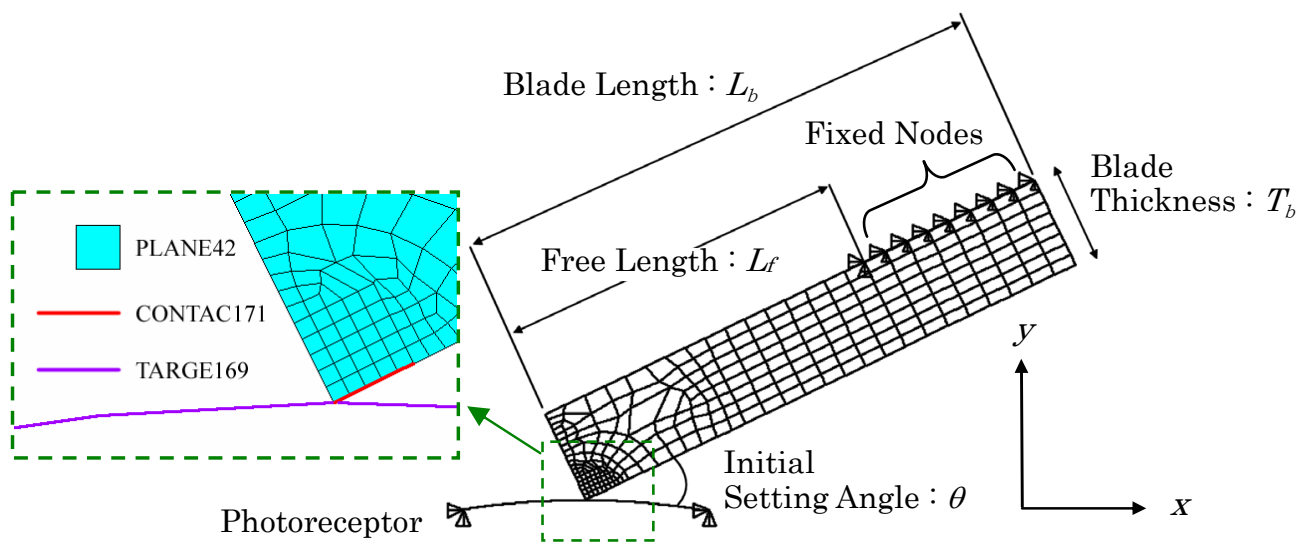

Fig. 3 Finite element model of the cleaning blade

Table 1 Design parameters of the cleaning blade

\begin{tabular}{lc}
\hline \multicolumn{1}{c}{ Parameters } & Values \\
\hline Blade Length $: L_{b}[\mathrm{~mm}]$ & 11.5 \\
Free Length $: L_{f}[\mathrm{~mm}]$ & 7.5 \\
Blade Thickness $: T_{b}[\mathrm{~mm}]$ & 2.0 \\
Initial Setting Angle $: \theta[$ degree] & 27.0 \\
Young's Modulus of Blade $[\mathrm{MPa}]$ & 5.88 \\
Density $\left[\mathrm{kg} / \mathrm{mm}^{3}\right]$ & $1.5 \times 10^{-6}$ \\
Poisson Ratio & 0.495 \\
Number of Nodes & 301 \\
\hline
\end{tabular}

\subsection{Formulation of a Coupled Mode via a Frictional Force ${ }^{(7)(8)}$}

A governing equation of the vibration of the finite element model shown in Fig. 3 can be described as

$$
[M]\{\ddot{X}\}+[K]\{X\}=\mu\left\{N_{F}\right\},
$$

where $\{X\}$ consists of displacement of node from equilibrium point, $[M]$ is mass matrix and $[K]$ is stiffness matrix, $\mu$ is a coefficient of friction, $\left\{N_{F}\right\}$ consists of normal force at contact points. Namely, the right hand side of Eq. (1) describes the frictional force applied to the edge of the blade.

According to the modal analysis theory, $\{X\}$ is represented with mass normalizing 
eigenfunctions $\left\{\phi_{i}\right\} \quad(\mathrm{i}=1, \ldots, \mathrm{n})$ as $\{X\}=[\Phi]\{q\}$, where $[\Phi]$ consists of $\left\{\phi_{i}\right\} \quad(\mathrm{i}=1, \ldots, \mathrm{n}), n$ is a number of mode, $\{q\}$ is a displacement vector in the modal space. Substituting $\{X\}=[\Phi]\{q\}$ into Eq. (1) leads to

$$
\{\ddot{q}\}+\left[\Omega^{2}\right]\{q\}=[\Phi]^{\mathrm{T}} \mu\left\{N_{F}\right\},
$$

where $[\Omega]$ is a diagonal matrix which consists of natural angle frequencies.

We assume that the normal force vector $\left\{N_{F}\right\}$ can be described by using the displacement vector $\{q\}$ as

$$
\left\{N_{F}\right\}=\left[\begin{array}{cccc}
\alpha_{11} & \alpha_{12} & \cdots & \alpha_{1 n} \\
\alpha_{21} & \alpha_{22} & \cdots & \alpha_{2 n} \\
\vdots & \vdots & \vdots & \vdots \\
\alpha_{N 1} & \alpha_{N 2} & \cdots & \alpha_{N n}
\end{array}\right]\left\{\begin{array}{c}
q_{1} \\
q_{2} \\
\vdots \\
q_{n}
\end{array}\right\}=[A]\{q\}
$$

where $[A]$ consists of contribution of a reaction force of each mode. Namely, the normal force $\left\{N_{F}\right\}$ can be also represented by the modal displacement $\{q\}$ as well as the physical displacement $\{X\}$. Substituting Eq. (3) into Eq. (2) leads to

$$
\{\ddot{q}\}+\left(\left[\Omega^{2}\right]-\mu[\Phi]^{\mathrm{T}}[A]\right)\{q\}=\{0\} .
$$

This is a basic formula of a coupled vibration via a frictional force in multi degrees of freedom model.

\section{Stability Analysis}

To begin with, a large deformation simulation that the blade pressed to the photoreceptor with interference $1.2 \mathrm{~mm}$ has been carried out. Next, an eigenvalue analysis of the deformed blade has been carried out. Table 2 shows the result of the eigenvalue analysis via ANSYS. The contribution to reaction force is obtained by using PRRSOL command of ANSYS. By substituting these values to Eq. (4), the eigenvalues are drawn as the root locus for the variation of friction coefficient (from 0 to 1.0) as shown in Fig. 4. With increasing of coefficient of friction, the 4 th mode and the 5th come close each other on the imaginary axis. Finally, the friction coefficient becomes larger $(\mu>0.44)$, these modes couple each other, eigenvalues of a coupled-modes move into the unstable area. Other eigenvalues (the 1 st, 2nd, 3rd and 6th) have stayed on the imaginary axis in the variation range of friction coefficient. This root locus reveals a typical argand diagram for coupled mode flutter of an undamped system via so-called Hamiltonian-Hopf bifurcation according to the classification by Paidoussis ${ }^{(9)}$.

Table 2 Results of eigenvalue analysis of the deformed blade via ANSYS

\begin{tabular}{cccc}
\hline Mode & $\begin{array}{c}\text { Natural } \\
\text { Frequency } \\
{[\mathrm{Hz}]}\end{array}$ & $\begin{array}{c}\text { Factor of Eigenvector } \\
\phi_{i 1}(i=1, \cdots, 6) \\
{\left[\mathrm{kg}^{-1 / 2}\right]}\end{array}$ & $\begin{array}{c}\text { Contribution to Reaction } \\
\text { Force } \\
\alpha_{1 i}(i=1, \cdots, 6) \\
{\left[\mathrm{N} \mathrm{m}^{-1} \mathrm{~kg}^{-1 / 2}\right]}\end{array}$ \\
\hline 1 & 1105 & -20.0 & -91972 \\
2 & 1979 & 324.3 & -116596 \\
3 & 2837 & 4.6 & -459827 \\
4 & 4431 & 95.9 & -542963 \\
5 & 4688 & -182.4 & -221179 \\
6 & 6040 & 278.5 & 256524 \\
\hline
\end{tabular}




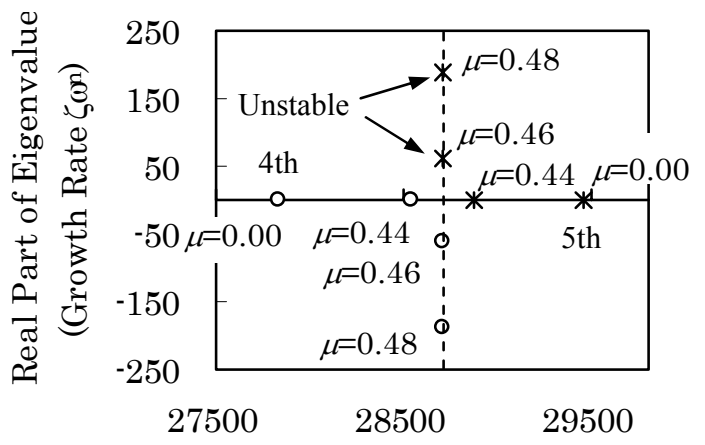

Imaginary Part of Eigenvalue

(Natural Frequency $\omega_{\mathrm{n}}$ )

Fig. 4 Root locus with the variation of friction coefficient

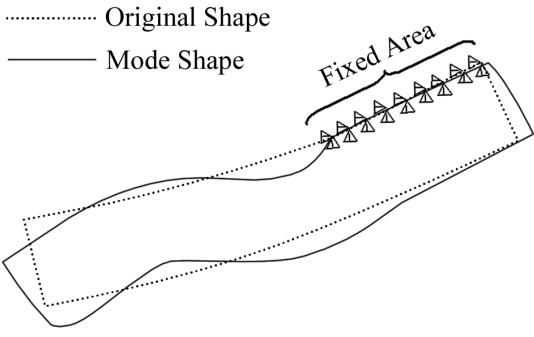

(a) 4th mode (bending)

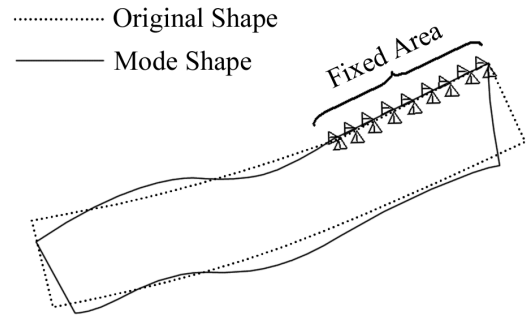

(b) 5th mode (shrinking)

Fig. 5 Mode shape of eigenfunction

What has to be noticed is that the imaginary part of the unstable mode is $28793 \mathrm{rad} / \mathrm{sec}$ i.e. the natural frequency is $4582 \mathrm{~Hz}$. This is good agreement with the frequency analysis result in an actual machine as indicated in Fig. 2.

Furthermore, as shown in Fig.5, we confirmed that the shape of eigenfunction of the 4th mode and the 5th mode are a bending and a shrinking, respectively.

From these results, we can conclude that this cleaning blade noise is caused by coupled-mode flutter between a bending mode and shrinking mode.

\section{Derivation of Equations Governing the Unstable Modes}

As discussed above, the coupling between the 4th and the 5th mode causes this self-excited vibration. Thus, we can focus our attention on these two modes. Here, from the result of the observation that the amplitude of this vibration converges to a non-trivial constant value, we can assume existence of nonlinear term and rewrite Eq. (4) as follows:

$$
\left\{\begin{array}{l}
\ddot{q}_{4} \\
\ddot{q}_{5}
\end{array}\right\}+\left[\begin{array}{cc}
\omega_{4}^{2}-\mu \phi_{41} \alpha_{14} & -\mu \phi_{41} \alpha_{15} \\
-\mu \phi_{51} \alpha_{14} & \omega_{5}^{2}-\mu \phi_{51} \alpha_{15}
\end{array}\right]\left\{\begin{array}{c}
q_{4} \\
q_{5}
\end{array}\right\}=\left\{\begin{array}{l}
f_{4} \\
f_{5}
\end{array}\right\},
$$

where $f_{i}(i=4,5)$ is unknown nonlinear component. By introducing a state vector $\{Z\} \equiv\left\{\begin{array}{llll}\dot{q}_{4} & \dot{q}_{5} & q_{4} & q_{5}\end{array}\right\}^{\mathrm{T}}$, we can rewrite Eq. (5) as

$$
[R]\{\dot{Z}\}+[\Gamma]\{Z\}=\left\{F_{z}\right\},
$$

where 
$[R] \equiv\left[\begin{array}{cccc}0 & 0 & 1 & 0 \\ 0 & 0 & 0 & 1 \\ 1 & 0 & 0 & 0 \\ 0 & 1 & 0 & 0\end{array}\right],[\Gamma] \equiv\left[\begin{array}{cccc}-1 & 0 & 0 & 0 \\ 0 & -1 & 0 & 0 \\ 0 & 0 & \omega_{4}^{2}-\mu \phi_{41} \alpha_{14} & -\mu \phi_{41} \alpha_{15} \\ 0 & 0 & -\mu \phi_{51} \alpha_{14} & \omega_{5}^{2}-\mu \phi_{51} \alpha_{15}\end{array}\right],\left\{F_{z}\right\} \equiv\left\{\begin{array}{llll}0 & 0 & f_{4} & \left.f_{5}\right\}^{\mathrm{T}} .\end{array}\right.$

A trial homogeneous solution of Eq. (6) is assumed to be $\{Z\}=\{\Psi\} \mathrm{e}^{\lambda t}$. By substituting this trial solution into Eq. (6), we can obtain

$$
\lambda[R]\{\Psi\}+[\Gamma]\{\Psi\}=\{0\} .
$$

The characteristic equation of the system described Eq. (7) is the second order algebraic equation for $\lambda^{2}$. If $\lambda^{2}$ has complex number, $\lambda$ consists of two couples of complex conjugate value that are located in symmetry about the imaginary axis. Namely, two of four eigenvalues must have a complex number with a positive real part. We now are interested only in these two eigenvalues to derive equations governing the unstable modes.

At this time, we have to face the problem that the orthogonality between eigenvectors $\{\Psi\}$ for each $\lambda$ is not established, because of the non-symmetricity of the matrix $[\Gamma]$. As a solution for this problem, we have extracted the unstable modes by the adjoint system. For more details, see Rousselet and Herrmann ${ }^{(3)(4)}$.

At first, we introduce the following adjoint system with vector $\left\{\Psi^{\prime}\right\}$ determined by the solvability condition ${ }^{(10)(11)}$ :

$$
\lambda\left\{{\overline{\Psi^{\prime}}}^{\}^{\mathrm{T}}}[R]+\left\{\overline{\Psi^{\prime}}\right\}^{\mathrm{T}}[\Gamma]=\{0\}^{\mathrm{T}} .\right.
$$

We will now consider the $i$ th mode of the original system and a pre multiplication of Eq. (7) by $\left\{\overline{\Psi_{j}^{\prime}}\right\}^{\mathrm{T}}$ leads to

$$
\lambda_{i}\left\{\overline{\Psi_{j}^{\prime}}\right\}^{\mathrm{T}}[R]\left\{\Psi_{i}\right\}+\left\{\overline{\Psi_{j}^{\prime}}\right\}^{\mathrm{T}}[\Gamma]\left\{\Psi_{i}\right\}=0 .
$$

Also, considering the $j$ th mode of the adjoint system and a post multiplication of Eq. (8) by $\left\{\Psi_{i}\right\}$ leads to

$$
\lambda_{j}\left\{\overline{\Psi_{j}^{\prime}}\right\}^{\mathrm{T}}[R]\left\{\Psi_{i}\right\}+\left\{\overline{\Psi_{j}^{\prime}}\right\}^{\mathrm{T}}[\Gamma]\left\{\Psi_{i}\right\}=0 .
$$

Subtraction between Eq. (9) and Eq. (10) conduces to

$$
\left(\lambda_{i}-\lambda_{j}\right)\left\{\overline{\Psi_{j}^{\prime}}\right\}^{\mathrm{T}}[R]\left\{\Psi_{i}\right\}=0 .
$$

If $i \neq j$, we can obtain the following relationship:

$$
\begin{aligned}
& \left\{\overline{\Psi_{j}^{\prime}}\right\}^{\mathrm{T}}[R]\left\{\Psi_{i}\right\}=0, \\
& \left\{\overline{\Psi_{j}^{\prime}}\right\}^{\mathrm{T}}[\Gamma]\left\{\Psi_{i}\right\}=0 .
\end{aligned}
$$

On the other hand, a trial solution of the Eq. (6) including the nonlinear component is assumed to be as

$$
\{Z\}=\sum_{k=1}^{4}\left\{\Psi_{k}\right\} \xi_{k}
$$


By substituting this trial solution into Eq. (6), followed by pre-multiplying the adjoint vector $\left\{\Psi^{\prime}\right\}$ and utilizing the relationship of Eq. (12), the decomposed mode can be described as

$$
\left\{{\overline{\Psi_{i}^{\prime}}}^{\prime}{ }^{\mathrm{T}}[R]\left\{\Psi_{i}\right\} \dot{\xi}_{i}-\lambda_{i}\left\langle\overline{\Psi_{i}^{\prime}}\right\}^{\mathrm{T}}[R]\left\{\Psi_{i}\right\} \xi_{i}=\left\{\bar{\Psi}_{i}^{\prime}\right\}^{\mathrm{T}}\left\{F_{z}\right\} \quad i=1, \cdots, 4 .\right.
$$

As mentioned above, we are interested only in the two unstable modes, whose eigenvalues have complex conjugate number. We describe these modes as $\lambda_{1}$ and $\bar{\lambda}_{1}$. Equation (14) therefore yields

$$
\begin{aligned}
& \Lambda_{1} \dot{\xi}_{1}-\lambda_{1} \Lambda_{1} \xi_{1}=\zeta_{1}, \\
& \Lambda_{1} \dot{\xi}_{1}-\bar{\lambda}_{1} \Lambda_{1} \bar{\xi}_{1}=\bar{\zeta}_{1},
\end{aligned}
$$

where $\Lambda_{1} \equiv\left\{\overline{\Psi_{1}^{\prime}}\right\}^{\mathrm{T}}[R]\left\{\Psi_{1}\right\}, \zeta_{1} \equiv\left\{\overline{\Psi_{1}^{\prime}}\right\}^{\mathrm{T}}\left\{F_{z}\right\}$. Moreover, the following new variables are introduced: $\xi_{1} \equiv q_{u}+\mathrm{i} q_{u i}, \lambda_{1} \equiv \lambda_{u r}+\mathrm{i} \lambda_{u i}, \zeta_{1} \equiv \zeta_{1 r}+\mathrm{i} \zeta_{1 i}$. From multiplication of the first equation and the second one in Eq. (15), we can obtain

$$
\ddot{q}_{u}-2 \lambda_{u r} \dot{q}_{u}+\left(\lambda_{u r}^{2}+\lambda_{u i}^{2}\right) q_{u}=\frac{1}{\Lambda_{1}}\left(\dot{\zeta}_{1 r}-\lambda_{u r} \zeta_{1 r}-\lambda_{u i} \zeta_{1 i}\right)
$$

On a supposition that the right hand side of Eq. (16) consists mainly an unknown nonlinear component of the frictional force between a cleaning blade and a photoreceptor, we assume that the nonlinear component can be described as a function of $\dot{q}_{u}$ and $q_{u}$, namely $f\left(q_{u}, \dot{q}_{u}\right)$. Here, for the negative and positive displacements that have the same size, $f\left(q_{u}, \dot{q}_{u}\right)$ also should have the same size but different direction. Thus, the nonlinear term is the third order one obtained by a Taylor expansion of $f\left(q_{u}, \dot{q}_{u}\right)$, i.e. $q_{u}^{3}, q_{u}^{2} \dot{q}_{u}, q_{u} \dot{q}_{u}^{2}, \dot{q}_{u}^{3}$. We will consider the complex amplitude equation lead from the condition of not causing secular term in the method of multiple scales. $q_{u}^{2} \dot{q}_{u}$ and $\dot{q}_{u}^{3}$ can be considered as a candidate of nonlinear term which satisfies such a condition. It is well known as the van der Pol's equation that the former is substituted in Eq. (16); the latter case is corresponding to the Rayleigh's equation. In this paper, $\dot{q}_{u}^{3}$ is adopted as the nonlinear component, because two equations are essentially equivalent. As a result, we can formulate a coupled vibration due to the unstable mode as

$$
\ddot{q}_{u}-2 \lambda_{u r} \dot{q}_{u}+\delta \dot{q}_{u}^{3}+\left(\lambda_{u r}^{2}+\lambda_{u i}^{2}\right) q_{u}=0,
$$

where $\delta$ is a coefficient of the nonlinear component. The detailed procedures of deciding $\delta$ will be mentioned later.

\section{Nonlinear Analysis}

\subsection{Nonlinear Solution}

We will solve Eq. (17) using the method of multiple scales. Here, dimensionless mode displacement $v_{u}$ and dimensionless time $\tau$ are defined as $v_{u} \equiv q_{u} / L, \tau \equiv t / T$, where $L=\sqrt{2 \lambda_{u r} / \delta \lambda_{u i}^{2}}, T=1 / \lambda_{u i}$. Since $\lambda_{u r}<<\lambda_{u i}$, we can say $\lambda_{u r}^{2}+\lambda_{u i}^{2} \approx \lambda_{u i}^{2}$, and rewrite Eq. (17) as follows:

$$
\frac{d^{2} v_{u}}{d \tau^{2}}-2 \varepsilon\left\{1-\left(\frac{d v_{u}}{d \tau}\right)^{2}\right\} \frac{d v_{u}}{d \tau}+v_{u}=0
$$

where $\varepsilon \equiv \lambda_{u r} / \lambda_{u i}$. We now assume that an approximate solution of Eq. (18) can be 
described with $\varepsilon$ as $v_{u}=v_{u 0}\left(\tau_{0}, \tau_{1}\right)+\varepsilon v_{u 1}\left(\tau_{0}, \tau_{1}\right)+\cdots$, where $\tau_{0} \equiv \tau, \tau_{1} \equiv \varepsilon \tau$. Assuming that $v_{u 0}=A\left(\tau_{1}\right) \mathrm{e}^{\mathrm{i} \tau_{0}}+$ C.C., in order to avoid the appearance of the no secular term of $\tau_{0}$, the complex amplitude $A\left(\tau_{1}\right)$ has to satisfy the following equation:

$$
D_{1} A=(1-3 A \bar{A}) A \text {. }
$$

Steady-state amplitude $A=A_{s}$ can be described as $A_{s} \bar{A}_{s}=1 / 3$, when the left hand side of Eq. (19) is replaced by 0 . Thus, we can obtain the nonlinear solution $v_{u}$ as

$$
v_{u}=\frac{2}{\sqrt{3}} \sin \tau+\mathrm{O}(\varepsilon) .
$$

\subsection{Reconstruction of Unstable Mode into 2-DOF System}

Here, we try to express $q_{4}$ and $q_{5}$ at the steady state by using the unstable mode $q_{\mathrm{u}}$ clarified in previous section, in order to express $\{X\}$ as $\{X\}=\left\{\phi_{4}\right\} q_{4}+\left\{\phi_{5}\right\} q_{5}$ by referring to Herrmann and Roussellet's method ${ }^{(3)(4)}$.

To start with, the nondimensionlization of Eq. (5) yields

$$
\left\{\begin{array}{l}
\ddot{v}_{4} \\
\ddot{v}_{5}
\end{array}\right\}+T^{2}\left[\begin{array}{cc}
\omega_{4}^{2}-\mu \phi_{41} \alpha_{14} & -\mu \phi_{41} \alpha_{15} \\
-\mu \phi_{51} \alpha_{14} & \omega_{5}^{2}-\mu \phi_{51} \alpha_{15}
\end{array}\right]\left\{\begin{array}{l}
v_{4} \\
v_{5}
\end{array}\right\}=\frac{T^{2}}{L}\left\{\begin{array}{l}
f_{4} \\
f_{5}
\end{array}\right\},
$$

where $v_{i} \equiv q_{i} / L \quad(i=4,5)$. At first, for the linear equation given by replacing of the right hand side of Eq. (21) with 0 , we introduce the following trial solution:

$$
\left\{\begin{array}{c}
v_{4} \\
v_{5}
\end{array}\right\}=\left\{\begin{array}{l}
A_{4}(\tau) \\
A_{5}(\tau)
\end{array}\right\} \mathrm{e}^{\mathrm{i} \tau}+\text { C.C. }
$$

where $A_{4}$ and $A_{5}$ are unknown complex amplitude, because the nonlinear component at the right hand side of Eq. (21) is necessary for determination of $A_{4}$ and $A_{5}$. At the present stage, we cannot determine $A_{4}$ and $A_{5}$, but the amplitude ratio can be obtained by substituting Eq. (22) into the linear part of Eq. (21) as follows:

$$
R \equiv \frac{A_{4}}{A_{5}}=\frac{-1+T^{2}\left(\omega_{5}^{2}-\mu \phi_{51} \alpha_{15}\right)}{\mu \phi_{51} \alpha_{14} T^{2}},
$$

where $R$ is the amplitude ratio of $A_{4}$ to $A_{5}$. Equation (22) is rewritten as

$$
\left\{\begin{array}{c}
v_{4} \\
v_{5}
\end{array}\right\}=\left\{\begin{array}{l}
R \\
1
\end{array}\right\} A_{5} \mathrm{e}^{\mathrm{i} \tau}+\text { C.C. . }
$$

On the other hand, if we have focused our attention only about an unstable eigenvalue i.e. $\lambda_{1}$ and $\bar{\lambda}_{1}$, Equation (13) can be rewritten as $\left\{Z_{u}\right\}=\left\{\Psi_{1}\right\} \xi_{1}+\left\{\bar{\Psi}_{1}\right\} \bar{\xi}_{1}$. Here, from the definition: $\{Z\} \equiv\left\{\begin{array}{llll}\dot{q}_{4} & \dot{q}_{5} & q_{4} & q_{5}\end{array}\right\}^{\mathrm{T}}$ and the fact: $\left\{\Psi_{1}\right\}=\left\{\lambda_{1} \psi_{41} \quad \lambda_{1} \psi_{51} \quad \psi_{41} \quad \psi_{51}\right\}^{\mathrm{T}}$, we can obtain a following equation:

$$
\left\{\begin{array}{l}
q_{4} \\
q_{5}
\end{array}\right\}=\left\{\begin{array}{l}
\psi_{41} \\
\psi_{51}
\end{array}\right\} \xi_{1}+\text { C.C. . }
$$

After nondimensionlization of Eq. (25), we can rewrite it by using $R$ as follows: 


$$
\left\{\begin{array}{l}
v_{4} \\
v_{5}
\end{array}\right\}=\left\{\begin{array}{l}
R \\
1
\end{array}\right\} \psi_{51} \frac{\xi_{1}^{*}}{L}+\text { C.C. }
$$

where $\xi_{1}^{*}$ is dimensionless of $\xi_{1}$. We can replace $\psi_{51} \xi_{1}^{*}$ with $\xi_{1}^{*}$, because the magnitude of $\xi_{1}^{*}$ has not been determined. Thus, Equation (26) can be rewritten as

$$
\left\{\begin{array}{l}
v_{4} \\
v_{5}
\end{array}\right\}=\left\{\begin{array}{l}
R \\
1
\end{array}\right\} \frac{\xi_{1}^{*}}{L}+\text { C.C. . }
$$

Comparison of Eq. (24) and Eq. (27) leads $\xi_{1}^{*} / L=A_{5} \mathrm{e}^{\mathrm{i} \tau}$. Furthermore, in view of $v_{u}=\operatorname{Re}\left\{\xi_{1}^{*} / L\right\}$, the following equation has been obtained:

$$
v_{u}=\left|A_{5}\right| \sin \left(\tau+\angle A_{5}\right) \text {, }
$$

where $\left|A_{5}\right|$ is the absolute value of $A_{5}, \angle A_{5}$ is the argument of $A_{5}$. Therefore, comparison of Eq. (28) and Eq. (20) leads $\left|A_{5}\right|=2 / \sqrt{3}$. From Eq. (24), it is clear that $v_{4}$ and $v_{5}$ can be described as

$$
\begin{aligned}
& v_{4}=2\left|A_{5}\right||R| \sin (\tau+\angle R), \\
& v_{5}=2\left|A_{5}\right| \sin \tau,
\end{aligned}
$$

where $|R|$ is the absolute value of $R, \angle R$ is the argument of $R$. By substituting the relationship: $\left|A_{5}\right|=2 / \sqrt{3}$ into Eq. (29) and dimensionlization of $v_{4}$ and $v_{5}$, we can express $q_{4}$ and $q_{5}$ at the steady state as follows:

$$
\begin{aligned}
& q_{4}(t)=\frac{4|R|}{\lambda_{u i}} \sqrt{\frac{2 \lambda_{u r}}{3 \delta}} \sin \left(\lambda_{u i} t+\angle R\right), \\
& q_{5}(t)=\frac{4}{\lambda_{u i}} \sqrt{\frac{2 \lambda_{u r}}{3 \delta}} \sin \lambda_{u i} t .
\end{aligned}
$$

\subsection{Formula of Steady-State Amplitude and Determination of $\delta$}

From what has been discussed above, the theoretical equation of steady-state amplitude can be described as follows:

$$
x_{\eta}=\phi_{4 \eta} q_{4}+\phi_{5 \eta} q_{5}=\frac{4}{\lambda_{u i}} \sqrt{\frac{2 \lambda_{u r}\left(a^{2}+b^{2}\right)}{3 \delta}} \sin \left(\lambda_{u i} t+\sigma\right),
$$

where $x_{\eta}$ is displacement at the node number $\eta, \phi_{i \eta}(i=4,5)$ is component of the eigenfunction at the node number $\eta, a=|R| \phi_{4 \eta} \cos \angle R+\phi_{5 \eta}, \quad b=|R| \phi_{4 \eta} \sin \angle R$, $\sigma=\tan ^{-1}(b / a)$ respectively. Furthermore, we assume that a measurement value of steady-state amplitude $x_{\text {exp }}$ is equivalent to the above theoretical value $x_{\eta}$ at the same point, the coefficient of nonlinear term $\delta$ can be described as

$$
\delta=\frac{32}{3} \frac{\lambda_{u r}}{\lambda_{u i}^{2} x_{e x p}^{2}}\left\{\left(|R| \phi_{4 \eta} \cos \angle R+\phi_{5 \eta}\right)^{2}+\left(|R| \phi_{4 \eta} \sin \angle R\right)^{2}\right\} .
$$

In order to determine nonlinear term $\delta$, the results of measurement are applied to Eq. (32) as follows. The frequency spectrum is shown in Fig. 2, The growth rate of the amplitude is approximated as shown in Fig. 6, respectively. 


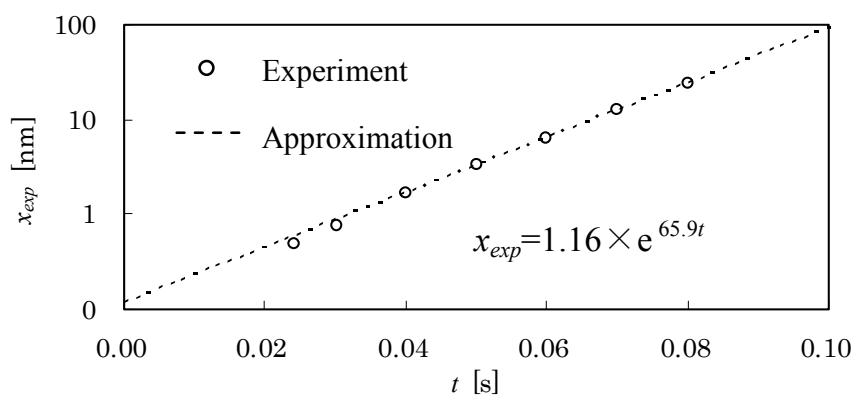

Fig. 6 Approximation of growth rate of the amplitude

From these results, we can obtain the values of $x_{e x p}, \lambda_{u i}, \lambda_{u r}$. Besides, $\phi_{41}, \phi_{5,1}$ are shown in Table 2. In order to determine $R$ by Eq. (23), we need contribution to reaction force $\alpha_{14}$, $\alpha_{15}$ and coefficient of friction $\mu$. The contribution to reaction force $\alpha_{14}$ and $\alpha_{15}$ are shown in Table 2. The coefficient of friction $\mu$ has been estimated as follows, because it is difficult to measure coefficient of friction accurately in an actual machine. The growth rate of this vibration is 65.9 as shown in Fig. 6. By applying this value to Fig. 4, it can be estimated that the coefficient of friction $\mu$ is 0.46 , when a growth rate is $65.9 . R$ is calculated as 0.51 from Eq. (23). Finally, Substituting of above values into Eq. (32) provides: $\delta=3.3 \times 10^{12}$.

\section{Example Case Studies}

\subsection{Numerical Studies}

To begin with, we consider three trial modification cases as follows: Case $1\left(T_{b}=1.6 \mathrm{~mm}\right.$, $\left.L_{f}=7.5 \mathrm{~mm}\right)$, Case2 $\left(T_{b}=2.2 \mathrm{~mm}, L_{f}=7.5 \mathrm{~mm}\right)$, Case $3\left(T_{b}=2.0 \mathrm{~mm}, L_{f}=6.4 \mathrm{~mm}\right)$. Here, design parameters except for the free length $L_{f}$ and thickness $T_{b}$ have the same values. Furthermore, we assume that value of friction coefficient $\mu$, growth rate $\lambda_{u r}$ and coefficient of nonlinear term $\delta$ are maintained after modification. Namely, $\mu=0.46, \lambda_{u r}=65.9$ and $\delta=3.3 \times 10^{12}$.

Eigenvalue analysis of these modified blades is carried out by ANSYS. Natural frequency, eigenfunction, and contribution of reaction force regarding the 4th and the 5th mode are shown in Table 3. After substituting these values into Eq. (4), natural frequency of coupled mode is calculated. Furthermore, steady-state amplitude is calculated from Eq. (31). Results of calculation are shown in Table 4.

Table 3 Results of eigenvalue analysis of the modified blade

\begin{tabular}{|c|c|c|c|c|}
\hline & & $\begin{array}{l}\text { Natural } \\
\text { Frequency } \\
{[\mathrm{Hz}]}\end{array}$ & $\begin{array}{c}\text { Factor of Eigenvector } \\
\qquad \begin{array}{c}\phi_{i 1}(i=4,5) \\
{\left[\mathrm{kg}^{-1 / 2}\right]}\end{array}\end{array}$ & $\begin{array}{l}\text { Contribution to Reaction Force } \\
\qquad \begin{array}{c}\alpha_{1 i}(i=4,5) \\
{\left[\mathrm{N} \mathrm{m}^{-1} \mathrm{~kg}^{-1 / 2}\right]}\end{array}\end{array}$ \\
\hline \multirow{2}{*}{ Case 1} & 4 & 4498 & 77.7 & $-6.6 \times 10^{5}$ \\
\hline & 5 & 5290 & -152.9 & $-2.3 \times 10^{5}$ \\
\hline \multirow{2}{*}{ Case 2} & 4 & 4334 & 147.0 & $-3.0 \times 10^{5}$ \\
\hline & 5 & 4511 & -109.8 & $-2.6 \times 10^{5}$ \\
\hline \multirow{2}{*}{ Case 3} & 4 & 4858 & 41.1 & $-2.2 \times 10^{5}$ \\
\hline & 5 & 5279 & -176.1 & $-5.8 \times 10^{5}$ \\
\hline
\end{tabular}

Table 4 Natural frequencies of coupled-mode and steady-state amplitude (results of calculation)

\begin{tabular}{lcc}
\hline & Natural Frequency $: \lambda_{u i}[\mathrm{~Hz}]$ & Steady-state Amplitude $: x_{\eta}[\mathrm{nm}]$ \\
\hline Case 1 & 4976 & 17.7 \\
Case 2 & 4419 & 45.8 \\
Case 3 & 4944 & 16.2 \\
\hline
\end{tabular}




\subsection{Experiment}

We confirm the validity of the calculation by the comparison with the experiment. An experimental apparatus is shown in Fig. 7. It consists of three main parts: a photoreceptor used in an actual machine, a drive motor that rotates the photoreceptor, two linear stages for adjustment of a relative position between the photoreceptor and the cleaning blade.

The experimental results are shown in Fig. 8. Besides, Fig. 9 summarizes the results of the calculation and the experiment in each case. Consequently, Fig. 9 shows that the calculation results are qualitatively in agreement with the experimental one about the variation in the amplitude and the frequency.

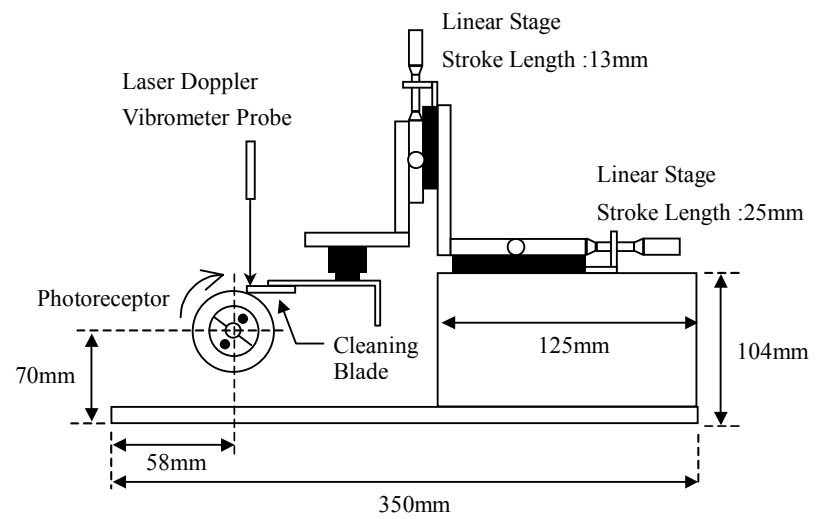

Fig. 7 Experimental apparatus for evaluation of modified blade

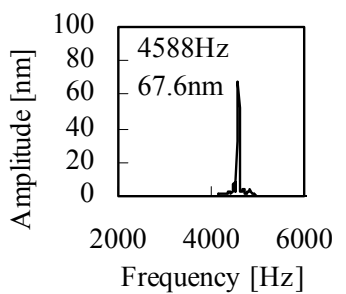

(a) Original

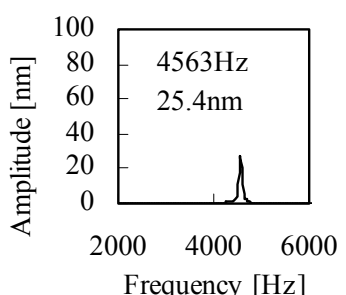

(c) Case 2

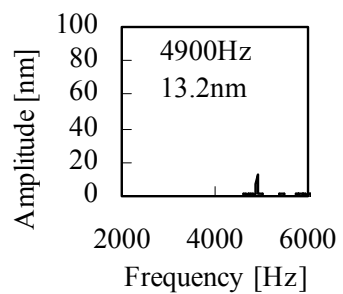

(b) Case 1

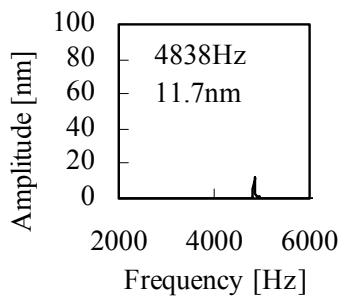

(d) Case 3

Fig. 8 Experimental results of vibration at the edge of blade in each case

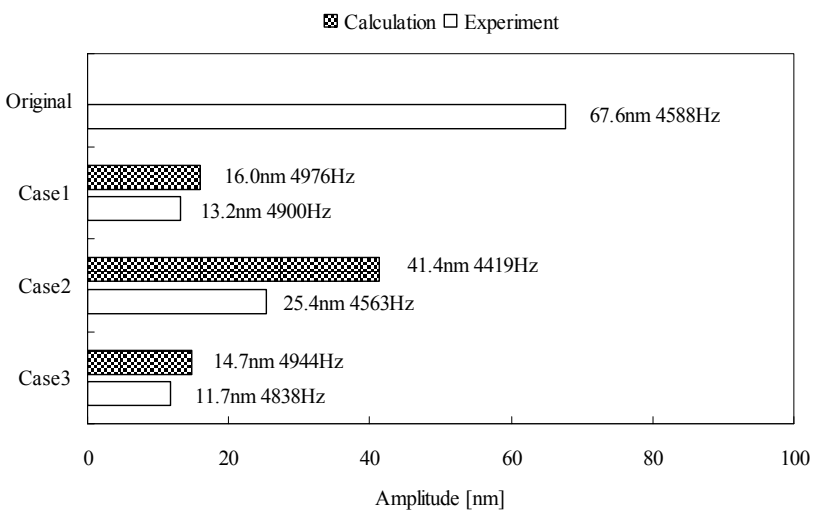

Fig. 9 Comparison between calculation and experiment about steady-state amplitude and frequency 


\section{Conclusion}

From the discussion about the mechanism of the self-excited vibration of the cleaning blade in a laser printer, we can obtain the following conclusions.

First, this self-excited vibration is caused by the coupling between a bending mode and a shrinking mode induced friction. The root locus of the system reveals a typical argand diagram for coupled mode flutter of an undamped system via so-called Hamiltonian-Hopf bifurcation.

Second, the nonlinear amplitude equation was derived by extracting the unstable modes with the adjoint vector instead of the eigenvector of the system. The nonlinear term was decided from the condition that the growth of the amplitude can be restrained referring to the Rayleigh's equation.

Third, referring to Hermann and Rousselet's method in pipes conveying fluid, we reconstructed the solution of unstable mode displacement obtained by the method of multiple scales to the 2-DOF system. The theoretical equation of steady-state amplitude was described with the eigenfunctions of the non-coupled system and the unstable mode displacement.

Finally, we confirmed that the calculation results were qualitatively agreement with experiment results about the variation in the amplitude and the frequency in some modification cases. This fact reveals clearly that our study has enough validity to explain the mechanism of the self-excited vibration.

\section{References}

(1) Nakamura,K., Generation Mechanism of Unusual Noise in the Laser-Beam Printer, Trans. Jpn. Soc. Mech. Eng., Series C, Vol.62,No.601(1996), pp.3428-3433.

(2) Kawamoto,H., Chatter Vibration of a Cleaner Blade in Electrophotography, Journal of Imaging Science and Technology, Vol.40-1 (1996), pp.8-13.

(3) Rousselet,J., Dynamic Behavior of Pipes Conveying Fluid Near Critical Velocities, (1975), $\mathrm{PhD}$ dissertation, Stanford University.

(4) Rousselet,J., Herrmann,G., Flutter of Articulated Pipes at Finite Amplitude, Journal of Applied Mechanics, Vol.44,No.1(1977), pp.154-158.

(5) Grenouillat,R., Leblanc,C., Simulation of Chatter Vibrations for Wiper Systems, SAE 2002 World Congress, 2002-01-1239(2002-3).

(6) Ono,K.,Suzuki,A., Analysis of Friction-Induces Self-Excited Vibration of a Contact Recording Head Slider Using a Two-Degrees-of-Freedom Model, Trans. Jpn. Soc. Mech. Eng., Series C, Vol.63,No.614(1997), pp.3343-3351.

(7) Kasama,M., Yoshizawa,M., Yu,Y., Itoh,T., Itoh,Y., Oscillation of a Cleaning Blade in a Laser Printer (1st Report, Observation of Phenomena in an Actual Machine and Consideration of Oscillation Mechanism), Trans. Jpn. Soc. Mech. Eng., Series C, Vol. 72,No.713 (2006), pp.76-83.

(8) Yu,Y., Analysis of Friction-Induced Vibrations of a Xerographic Cleaning Blade, Journal of Imaging Science and Technology, Vol.50-1 (2006), pp.103-110.

(9) Paidoussis,M.P., Fluid Structure Interactions Vol.1,( 1998), Academic Press.

(10) Nayfeh,A.H., Perturbation Methods, (1973), Weily \& Sans.

(11) Nayfeh,A.H., Introduction to Perturbation Techniques, (1981), Weily \& Sans.

(12) Kasama,M., Yoshizawa,M., Yu,Y., Itoh,T., Itoh,Y., Oscillation of a Cleaning Blade in a Laser Printer (2nd Report, Introduction of Nonlinear Amplitude Equation of Unstable Vibration via a Coupled Mode Flutter), Trans. Jpn. Soc. Mech. Eng., Series C, Vol. 72,No.724 (2006), pp.3719-3727. 